\title{
ON A RELATIONSHIP BETWEEN RECORD VALUES AND ROSS'S MODEL OF ALGORITHM EFFICIENCY
}

\author{
DIETMAR PFEIFER, ${ }^{*}$ Technical University Aachen
}

Recently Ross ((1981), (1983), Chapter 4.6) has developed a simple Markov chain model for an average-case analysis of the simplex algorithm in linear programming. Characteristically, this algorithm moves through the extreme points of the feasible region in such a way that only those points are successively considered which improve the actual value of the gain function (see e.g. Hadley (1962)). If we assume the $N$ (say) extreme points to be arranged in such a way that the first point gives the largest and the $N$ th point the smallest value of the gain function, then the steps of the algorithm can appropriately be described by a finite Markov chain $S_{1}, \cdots, S_{N}$ with state space $\{1, \cdots, N\}$ such that

(1) $P\left(S_{1}=k\right)=\frac{1}{N}, \quad 1 \leqq k \leqq N \quad$ and $\quad P\left(S_{n+1}=k \mid S_{n}=i\right)=\frac{1}{i-1}, \quad 1 \leqq k<i \leqq N$

with 1 being an absorbing state. For this model Ross (1981), (1983) has shown that if $T_{N}$ denotes the number of steps required to reach state 1 for the first time then $T_{N}$ is approximately (for large $N$ ) Poisson distributed over $\mathbb{N}$ with mean $\log N$. Here we shall demonstrate that this result can also be obtained by record value theory. In fact, if $\left\{X_{n} ; n \in \mathbb{N}\right\}$ is an i.i.d. sequence of random variables following a uniform distribution over $\{1, \cdots, N\}$, then $\left\{S_{n} ; 1 \leqq n \leqq N\right\}$ is identically distributed with the lower record value sequence $\left\{X_{U_{n}} ; 1 \leqq n \leqq N\right\}$ where

$$
U_{1}=1, \quad U_{n+1}= \begin{cases}\min \left\{k ; X_{k}<X_{U_{n}}\right\} & \text { if } X_{U_{n}}>1, \\ U_{n}, & \text { otherwise. }\end{cases}
$$

This follows readily by arguments as in Shorrock (1972). Especially, $T_{N}$ is identically distributed with $T=\min \left\{n ; X_{U_{n}}=1\right\}$.

Unfortunately, distribution theory for records from discrete distributions is rather cumbersome; however, to obtain the asymptotic results as indicated, we can use a continuous approximation in the following way. Obviously, nothing is seriously changed if we assume the random variables $\left\{X_{n} ; n \in \mathbb{N}\right\}$ to be uniformly distributed over $\{1 / N, \cdots,(N-1) / N, 1\}$ except that now $T=\min \left\{n ; X_{U_{n}}=1 / N\right\}=\min \left\{n ; X_{U_{n}}<2 / N\right\}$. But for large $N$, we may approximately assume the $X_{n}$ 's to be uniformly distributed over the unit interval; then $T$ is close to the stopping time $T^{*}=\min \left\{n ; X_{U_{n}}<2 / N\right\}$ where now $\left\{U_{n} ; n \in \mathbb{N}\right\}$ is the associated record time sequence. But as is known from record value theory (see Shorrock (1972)), $\left\{-\log X_{U_{n}} ; n \in \mathbb{N}\right\}$ forms the arrival time sequence of a unit-rate Poisson process implying that $T^{*}$ follows exactly a Poisson distribution with mean $\log N+1-\log 2 \approx \log N$. This gives the desired result. Moreover, the above arguments suggest that for the original Markov chain $\left\{S_{1}, \cdots, S_{N}\right\}$ and large

Received 12 October 1984; revision received 30 January 1985.

* Postal address: Institut für Statistik und Wirtschaftsmathematik, RWTH Aachen, Wüllnerstrasse 3, D-5100 Aachen, W. Germany. 
$N\left\{-\log S_{n} / N ; 1 \leqq n \leqq N\right\}$ behaves approximately as the first $N$ arrival times $Z_{1}, \cdots, Z_{N}$ of a unit rate Poisson process, or equivalently,

$$
S_{n} \approx \operatorname{int}\left(N \exp \left(-Z_{n}\right)\right)+1,1 \leqq n \leqq N .
$$

\section{References}

Hadley, G. (1962) Linear Programming. Addison-Wesley, Reading, Mass.

Ross, S. M. (1981) A simple heuristic approach to simplex efficiency. Operations Research Center Report, University of California, Berkeley.

Ross, S. M. (1983) Stochastic Processes. Wiley, New York.

SHORROCK, R. W. (1972) On record values and record times. J. Appl. Prob. 9, 316-326. 